\title{
Electrical and Morphological Properties of Low Resistivity Mo thin Films Prepared by Magnetron Sputtering
}

\author{
G. Gordillo, F. Mesa, and C. Calderón \\ Departamento de Física, Universidad Nacional de Colombia, Bogotá, Colombia
}

Received on 8 December, 2005

\begin{abstract}
Mo thin films have been deposited using a DC magnetron sputtering system with an S-gun configuration electrode and characterized electrically and morphologically. The influence of the sputtering gas pressure and glow discharge (GD) power, on the electrical resistivity of Mo thin films and on the contact resistivity of Mo to $\mathrm{Cu}(\mathrm{In}, \mathrm{Ga}) \mathrm{Se}_{2}$ (CIGS) films was determined through an exhaustive parameter study. This study also allowed us to find the conditions to deposit Mo films with suitable properties for its use as back contact of solar cells based on CIGS. Resistivities smaller than $1 \times 10^{-4} \Omega . \mathrm{cm}$ and contact resistivities smaller than $0.3 \Omega \mathrm{cm}^{2}$ were found. Mo films with these characteristics are suitable for back contacts in solar cells based on CIGS. It was also found that the Mo thin films, deposited by DC magnetron sputtering on CIGS thin films, act effectively as ohmic contacts. The main contribution of this work was to obtain Mo thin films with adequate properties to be used as back contact for CIGS based solar cells using a DC sputtering system with S-gun configuration electrode, which allows growing the film with better surface quality and at a higher deposition rate than those deposited using the conventional planar RF sputtering system.
\end{abstract}

Keywords: Mo; Thin films; Solar cells; CIGS; Low resistivity

\section{INTRODUCTION}

Several metals, $\mathrm{Pt}, \mathrm{Au}, \mathrm{Ag}, \mathrm{Cu}$ and $\mathrm{Mo}$, have been investigated for using as electrical contact of $\mathrm{CuInSe}_{2}$ (CIS) and its gallium alloy (CIGS)-based solar cells [1-3]. Mo emerged as the dominant choice for back contact layer of CIS and CIGS solar cells because of its relative stability at the processing temperature, resistance to alloying with $\mathrm{Cu}$ and $\mathrm{In}$, and its low contact resistance to CIGS. Efficiencies greater than 19\% have been reported for this type of devices fabricated using Mo films, deposited by conventional rf magnetron sputtering, as ohmic back contact [4,5].

Some authors [6,7] have reported that a Schottky barrier is formed when Mo films are inadequately deposited on CIGS films. When a Schottky barrier is formed at the CIGS/Mo contact, substantial resistive losses occur, affecting negatively the device efficiency.

In this work we investigate how deposition parameters, such as pressure of the sputtering gas and power supplied to the sputtering system, influence the conductivity of the molybdenum films and the contact resistivity of the Mo/CIGS system. Through an exhaustive parameter study, conditions were found to get Mo films, which form ohmic contact on CIGS films.

\section{EXPERIMENTAL}

Molybdenum films were prepared using a DC magnetron sputtering system with an S-gun configuration electrode. The main difference of this system with the conventional planar rf sputtering systems, is that the S-gun configuration employs a central anode surrounded by an Mo cathode $99.9 \%$ pure, which presents a conic shaped concavity. Fig. 1 shows a diagram of the sputtering system used to prepare the molybdenum films. In this system the films are deposited outside the plasma region which allows growing them with better surface quality than using the sputtering system with planar electrode configuration. On the other hand, the deposition rate of the films grown using the system with S-gun configuration is significantly greater than those deposited using the conventional system with planar electrode configuration.

The deposition chamber was evacuated by a turbomolecular pump to a base pressure of $10^{-5}$ mbar and the Arpartial pressure was electronically controlled, using an automatic control system consisting of a Balzers RV6 040 regulating unit and an RME 010 regulating valve. Table I lists the deposition parameters of the molybdenum films and their ranges of variation.

The CIGS films were deposited through a chemical reaction of the precursor species, which were evaporated sequentially in a three-stage process [8]. In the first stage the elements In, $\mathrm{Ga}$ and $\mathrm{Se}$ are evaporated simultaneously on a substrate heated at $300^{\circ} \mathrm{C}$. During this stage a thin film of the compound (In, Ga) $)_{x} \mathrm{Se}_{y}$ is formed. In the second stage, $\mathrm{Cu}$ and Se are simultaneously deposited on the layer formed in the previous stage, keeping the substrate temperature at $600^{\circ} \mathrm{C}$. During the second stage the compound $\mathrm{Cu}(\mathrm{In}, \mathrm{Ga}) \mathrm{Se}_{2}$ is predominantly formed. However, secondary phases of $\mathrm{Cu}$ and In selenides are also formed. Finally, in a third stage In, Ga and $\mathrm{Se}$ are simultaneously evaporated at a substrate temperature of $600^{\circ} \mathrm{C}$ (at lower rates than in the first stage), in order to optimize the chemical composition of the CIGS films. The deposition process of the CIGS films is controlled with a thickness monitor (Maxtec TM-400), which uses a quartz crystal as sensor.

The film conductivity was measured using a standard fourpoint probe method to determine the resistance, and a Dektak profilometer was used to determine the thickness. The contact resistance $R_{C}$ of Mo on p-CIGS thin films was determined using the three-point method described elsewhere $[9,10]$. Variation of the current through contacts yields the I-V characteris- 


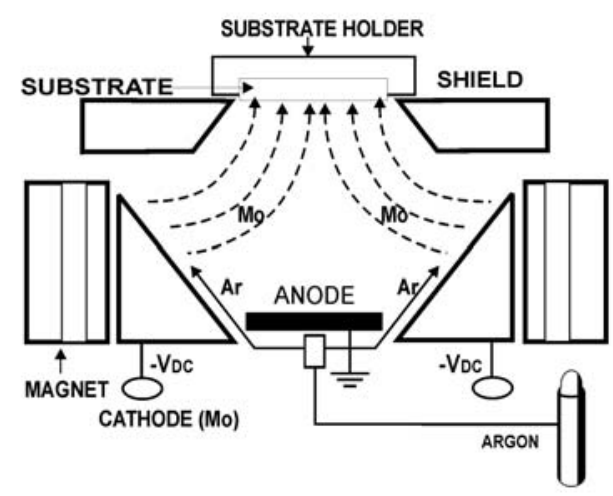

FIG. 1: Schematic diagram of the sputtering system with S-gun configuration electrode.

TABLE I: List of the deposition parameters and corresponding variation ranges, used to deposit the Mo thin films.

\begin{tabular}{|l|l|}
\hline Parameters & Variation range \\
\hline Substrate temperature & Room temperature \\
\hline Glow discharge power (W) & $50-200$ \\
\hline Sputtering current (mA) & $600-700$ \\
\hline Ar- partial pressure (mbar) & $1.1 \times 10^{-2}-1.6 \times 10^{-2}$ \\
\hline Bias voltaje (V) & $30-80$ \\
\hline Deposition time (min) & $6-26$ \\
\hline
\end{tabular}

tic and contact resistance $R_{C}$. For ohmic contacts, the contact resistivity $R_{C}$, defined as [9]

$$
\rho_{C}=\left.\frac{d V}{d J}\right|_{V=0}=R_{C} A_{C}\left(\Omega \mathrm{cm}^{2}\right)
$$

can be obtained from the slope of the J-V curve at zero bias, where $A_{C}$ is the contact area. The study of the morphological properties was carried out with a Park Scientific AFM microscope.

\section{RESULTS AND DISCUSSION}

\section{A. Electrical and morphological properties}

The effect of the working gas pressure and the glow discharge power on the electrical resistivity was studied in an attempt to find the deposition conditions to get low resistive Mo thin films. For that, Mo thin films were deposited varying the pressure from $1 \times 10^{-2}$ to $1.6 \times 10^{-2}$ mbar and the glow discharge (GD) power from 50 to $200 \mathrm{~W}$. The results indicated that the resistivity of the Mo films decreases by increasing the GD-power or by decreasing the Ar-pressure (see Fig. 3). On the other hand, measurements of the Mo films grain size, carried out with an AFM microscope, showed that this parameter increases by both, decreasing the Ar-pressure and increasing the GD-power. This result indicates that the reduction of the resistivity could be attributed, in part, to an increment of the grain size. In fig. 2 are depicted AFM images showing the influence of the working gas pressure and GD-power on the morphology of the Mo films.

The AFM studies showed clearly that the Ar-pressure and the GD-power affect the morphology of Mo films deposited by DC magnetron sputtering (with configuration S-Gun). In Fig. 3 are depicted curves that show the effect of the Arpressure and the GD-power on both, the electrical resistivity of Mo films and the grain size.

It is observed, correlating the curves of resistivity with those of grain size, that the decrease in resistivity of the Mo films is accompanied by an increase of the grain size. This behavior could be explained by assuming that increasing the grain size increases the carrier mobility of the Mo films. It seems that the increase of the grain size of the Mo films induces a reduction of the grain boundary potential barrier's height, as well as the number of grain boundaries the carrier has to cross during electrical transport, which, in turn, leads to a decrease of the resistivity.

Figure 4 shows results indicating that increasing the film thickness can also reduce the resistivity of the Mo-films. This behavior could be also attributed to an increase of the carrier mobility induced by increasing the grain size.

The lowest resistivity value we found for the Mo films was $9 \times 10^{-6} \Omega \mathrm{cm}$. This was obtained with Mo films prepared at Ar-pressure of $1.15 \times 10^{-2}$ mbar and a target power of $200 \mathrm{~W}$. Values of $1.6 \times 10^{-5} \Omega \mathrm{cm}$ have been reported [6] for Mo-films deposited on soda-lime glass substrates by planar RF magnetron sputtering and of $1.1 \times 10^{-5} \Omega \mathrm{cm}$ for Mo-films deposited by planar DC magnetron sputtering.

\section{B. Contact resistivity of Mo to $\mathrm{Cu}(\mathrm{In}, \mathrm{Ga}) \mathrm{Se}_{2}$ films}

The Mo films will be used as back contact layers for the $\mathrm{Cu}(\mathrm{In}, \mathrm{Ga}) \mathrm{Se}_{2}$ based solar cells, and the best parameter to characterize the quality of an ohmic contact is its contact resistivity $\rho_{C}$ [11]. So, the influence of the chemical composition (Ga-content) on the contact resistivity of Mo to $\mathrm{CuIn}_{x} \mathrm{Ga}_{1-x} \mathrm{Se}_{2}$ thin films was also studied.

Figure 5 depicts a curve of the contact resistivity of Mo on $\mathrm{CuIn}_{x} \mathrm{Ga}_{1-x} \mathrm{Se}_{2}$ thin films as a function of the chemical composition x. In Fig. 5 are shown curves of I vs. V, carried out to judge if the contact of $\mathrm{Mo}$ to $\mathrm{Cu}(\mathrm{In}, \mathrm{Ga}) \mathrm{Se}_{2}$ thin films is ohmic. The Mo films were deposited on the $\mathrm{Cu}(\mathrm{In}, \mathrm{Ga}) \mathrm{Se}_{2}$ films using the deposition parameters leading to the lowest resistivity value $\left(1.15 \times 10^{-2}\right.$ mbar and $\left.200 \mathrm{~W}\right)$.

The results of Fig. 5 indicate that the contact resistivity of Mo to $\mathrm{CuIn}_{x} \mathrm{Ga}_{1-x} \mathrm{Se}_{2}$ thin films increases by increasing the $\mathrm{Ga}$ content. However, the values of $\rho_{C}$ obtained in this work were lower than $0.3 \Omega \mathrm{cm}^{2}$, which is quite acceptable for ohmic contacts in solar cells. The linear behavior of the I-V curves confirms its ohmic character.

The SEM micrograph showed in Fig. 5b corresponds to the cross section of a typical solar cell, with structure $\mathrm{Mo} / \mathrm{CIGS} / \mathrm{CdS} / \mathrm{ZnO}$, we fabricate routinely in our laboratory, 

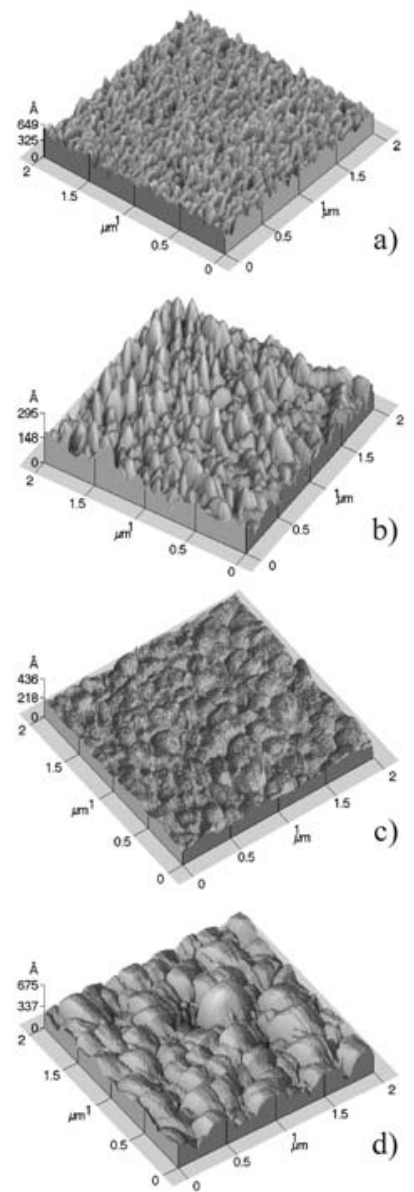

FIG. 2: Typical AFM images of Mo thin films, showing the effect of the Ar-partial pressure and of the GD-power on the morphology. a) $\mathrm{P}=100 \mathrm{~W}, \mathrm{Ar}-\mathrm{p}=1.6 \times 10^{-2} \mathrm{mbar}$ b) $\mathrm{P}=100 \mathrm{~W}, \mathrm{Ar}-\mathrm{p}=1.15 \times 10^{-2} \mathrm{mbar}$

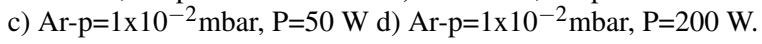

with efficiencies close to $9 \%$ [12].

\section{CONCLUSIONS}

Molybdenum thin films with adequate properties to be used as electrical back contact of solar cells based on CIGS were grown using a DC magnetron sputtering system with an S-gun configuration electrode. The results indicated that it is possible to get low resistivity Mo films by decreasing the Ar partial pressure and increasing the power of the glow discharge. This behavior is mainly attributed to an increase of the film grain size. The lowest value obtained for the resistivity was $9 \times 10^{-6} \Omega \mathrm{cm}$, which is lower than those reported in the literature for Mo films deposited by other methods. It was also found that the contact resistivity $\rho_{C}$ of the Mo/CIGS system is increased by increasing the Ga content; however, the values of $\rho_{C}$ we obtained for the CIGS films were lower than $0.3 \Omega \mathrm{cm}^{2}$; these values of $\rho_{C}$ are quite acceptable for ohmic contacts of
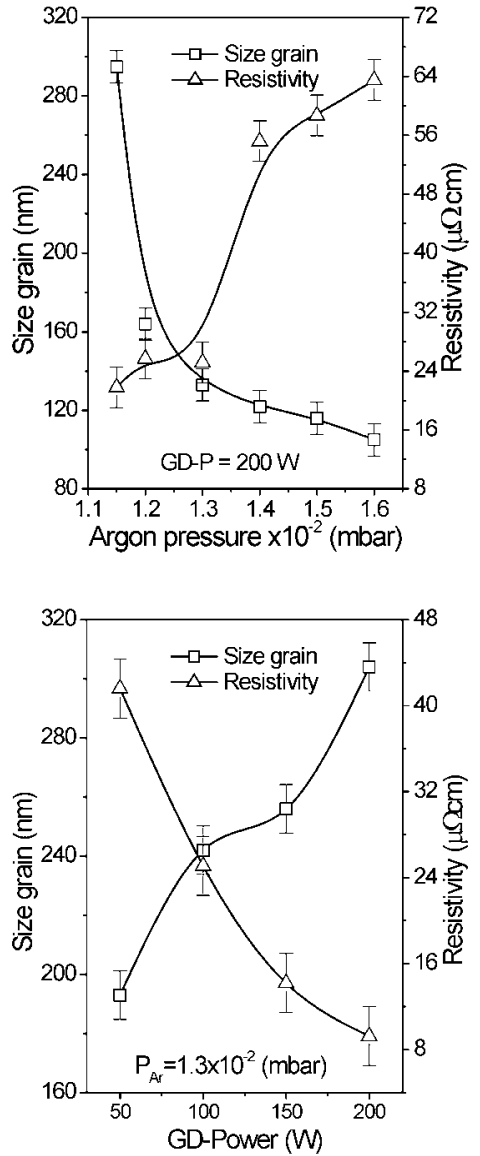

FIG. 3: Variation of the resistivity and grain size of Mo films as a function of a) Ar-pressure and b) GD-power.

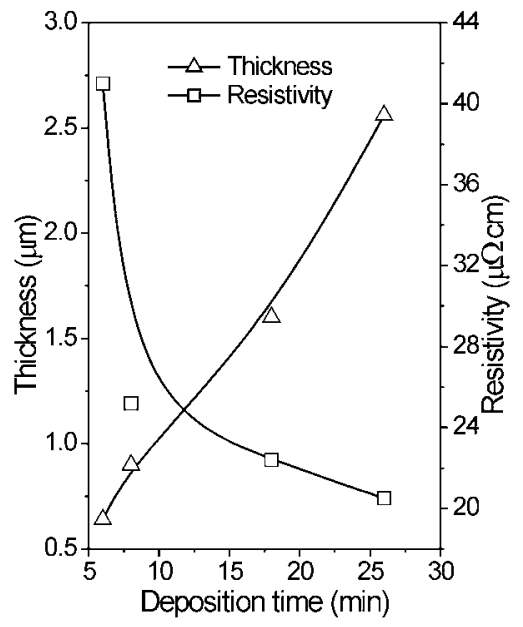

FIG. 4: Variation of the resistivity and Mo thin film thickness as a function of the deposition time. 


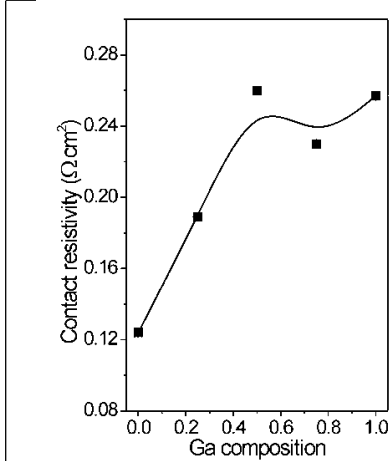

a)

b)
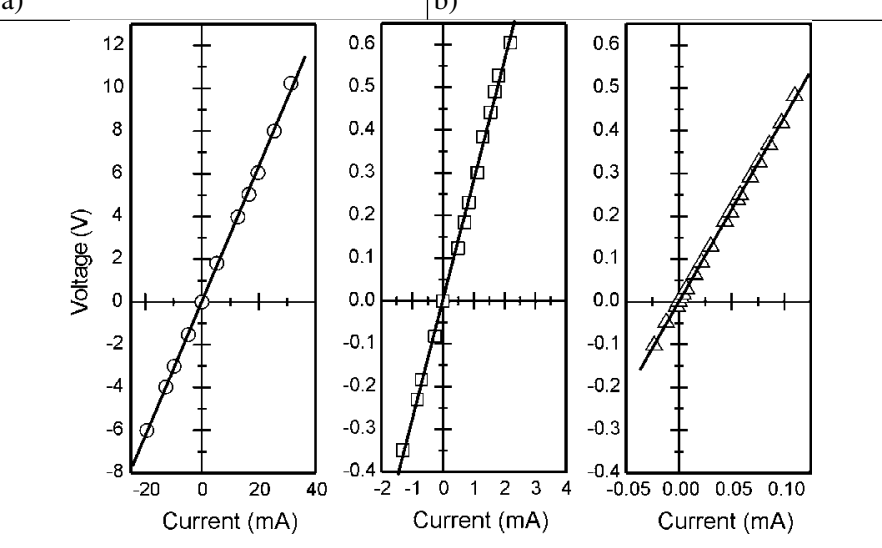

c)

FIG. 5.a) Variation of the contact resistivity of Mo to $\mathrm{CuIn}_{x} \mathrm{Ga}_{1-x} \mathrm{Se}_{2}$ thin films as a function of the chemical composition ( $x$ value), b) SEM micrograph of the cross section of a solar cell with structure Mo/CIGS/ZnO and c) curves of $\mathrm{I}$ vs. $\mathrm{V}$ of the system $\mathrm{Mo} / \mathrm{CuIn}_{x} \mathrm{Ga}_{1-x} \mathrm{Se}_{2}$, for $\mathrm{x}=0,0.5$ and 1 respectively.

solar cells operating at small solar concentration ratios. I vs. $\mathrm{V}$ measurements carried out with the Mo/CIGS/Mo system indicated a linear behavior, confirming the ohmic character of Mo on CIGS films.

\section{Acknowledgements}

The Universidad Nacional de Colombia and Colciencias supported this work.
[1] J.H. Scofield, A. Duda, D. Albin, B. L. Ballard and P. K. Predecki, Thin Solid Films 260, 26 (1995).

[2] R. J. Matson, O. Jamjoum, A. D. Buonaquisti, P. E. Russell et al., Solar Cells 11, 301 (1984).

[3] E. Moons, T. Engelhard and D. Cahen, J. Electron. Mater. 22 (3), 275 (1993).

[4] L.L. Kazmerski, Journal of Electron Spectroscopy and Related Phenomena 150, 105 (2006).

[5] K. Ramanathan, M. Contreras, I. Perkins, S Asher et. al., Progress in Photovoltaics: Res. Appl. 11, 225 (2003).

[6] W. Jaegaermann, T. Lher, C. Pettenkofer, Cryst. Res. Technol. 31, 273 (1996).

[7] T. Wada, N. Kohara, S. Nishiwaki and T. Negami, Thin Solid Films 387, 118 (2001).
[8] M.A. Contreras, B. Egaas, K. Ramanathan, J. Hiltner, A. Zwartlander, F. S. Hasoon, and R. Nuofi, Progress in Photovoltaics: Research and applications, 7, 311 (1999).

[9] A. L. Fahrenbruch, R. H. Bube, Fundamentals of Solar Cells, Academic Press, New York, (1992).

[10] T.C. Anthony, A.L. Fahrenbruch, and R. Bube, J. Electronic Mater. 11, 89 (1982).

[11] A. Musa, J. P. Ponpon, J. J. Grob, M. Hage-Ali et al., J. Appl. Phys. 54 (6), 3260 (1983).

[12] G. Gordillo, C. Calderón, and C. Quiñonez, 3rd World Conference on Photovoltaic Energy Conversion, Osaka, Japan (2003) 2P-D3-49. 